www.jmscr.igmpublication.org

Impact Factor 5.84

Index Copernicus Value: 71.58

ISSN (e)-2347-176x ISSN (p) 2455-0450

crossref DOI: _https://dx.doi.org/10.18535/jmscr/v5i9.164

\title{
Patterns of Oral Cancer Presentation in India - A Study of 1484 Patients
}

\author{
Authors \\ Dr Sanjoy Panda ${ }^{1}$, Dr Manjunath $\mathbf{N} M \mathbf{L}^{2}$ \\ ${ }^{1}$ Assistant Professor, Department of Head and Neck Surgery, RCC, Cuttack \\ ${ }^{2}$ Consultant, Department of Surg, HCG PCH, Cuttack \\ Email:namulimanju@yahoo.co.in, Mobile: 9013098006
}

\section{Introduction}

Oral cancer is the most common cancer in India. Geographical variations, human behavioral factors and genetic predisposition play a role in modifying the disease incidence, morphology, treatment response and prognosis. Apart from the tumor related factors, socioeconomic factors of an individual patient, primary residence of the patient and the country of origin add to the changes in the presentation and ultimate prognosis of the patient. Advantages of knowing the regional differences include, understanding the etiological factors, knowing the pathogenesis and disease biology, and ultimately targeting a particular population for early detection and behavioral modifications. Present study, a retrospective analysis of a prospectively maintained data, portrays the epidemiological features of patients with oral cancer presented to private comprehensive cancer care hospital in a resource constrained state of India.

\section{Methods}

Data of all the patients diagnosed with oral cavity squamous cell carcinoma was collected from the database of two major centers. Patients registered from January 2014 to June 2017 were included for the study. At presentation, the treating oncologist evaluated the patients with a thorough oral cavity and neck examination followed by necessary investigations. The treatment protocols and details are beyond the scope of the present article. The data collected was analyzed in Microsoft excel soft ware.

\section{Results}

A total of 1484 patients were considered for the analyses, managed between January 2014 and June 2017. Among 1484 patients, 22\% (n, 326) were females and $78 \%(n, 1158)$ were males. Mean age among the present cohort of patients was 49 years with age group of 40 to 55 years being most common. Eighty six percent of patients gave history of either pan or tobacco or both chewing. Smoking was prevalent among $23 \%$ of males and none of the females had alcohol or smoking tobacco exposure. Majority of the patients $(67 \%)$ presented with locally advanced cancers with left sided tumors being more common $(61 \%)$. As far as the site of involvement was concerned, buccal mucosa and alveobuccal sulcus regions (quid bed) were the most common sites $(48 \%)$ followed by tongue $(21 \%)$ and other sub-sites. 


\section{Discussion}

In the resource-constrained situation, management of patients needs to be tailored to the patient's needs and expectationswith a balanceto the available resources. In the same way generating high level of evidences by research in the form of randomised controlled trial and molecular studies is not possible. Resources need to be channelized more towards patient management than for the research. The options for an oncologist to formulate protocols in these situations, include extrapolating the results of research done elsewhere or choose a research methodology which consumes least resources. In this article epidemiological features of patients with oral cancer were recorded and studied retrospectively. Meaningful conclusions could be drawn from the results. These conclusions should be taken as starting points for the future research.

Oral cancer is the most common cancer in India ${ }^{(1)}$ due to excessive habit of smokeless tobacco in different forms. Age of presentation in any oncological research is important in terms of etiology, disease biology, management, prognosis and also implications on the resources of the family or region where the patient resides. Younger population suffers more with respect to oral cancer in India. As shown in our study oral cancer can affect patients in their second decade.
Mean age in our study was 49 years which is $10-$ 15 years lesser than most of the western studies $(2,3)$. In India, the oral cancer patients present in young age as tobacco habit initiates around 15-20 years of age in contrast to the Western world.

Tobacco chewing starts mostly by observing the family members, friends and peers ${ }^{(4)}$. Lack of awareness is not the reason for continuing the habit. Addiction liability of tobacco and social beliefs play a major role. Management of oral cancer in these patients is easier in terms of their general condition for treatment tolerance. Major issue is obtaining the resources for the management. Most of the time, the patient is the sole earning member and family does not have adequate financial reserves or insurances for the management. This issue should be highlighted in any awareness programs so that the members of the risk group should themselves take the responsibility.

The gender difference (male: female 4:1) of oral cancer incidence has important implications. Male preponderance is observed in the countries where tobacco and betel quid usage is rampant ${ }^{(5,6)}$. The possible cause for this discrepancy includes, the differential habit of using tobacco in different forms. Females use products, which are betel leaves based as shown in the table 1 .

Table 1: Showing commonly used products kept as quid or chewed in India

\begin{tabular}{|l|c|c|c|c|c|c|}
\hline Name of product & Tobacco & Pan leaves & Arecanut & Lime & Spices & Others \\
\hline Betel quid & - & + & + & + & $+/-$ & $+/-$ \\
\hline Betel tobacco quid & + & + & + & + & $+/-$ & $+/-$ \\
\hline Gopal/gundi & + & - & + & + & - & - \\
\hline Gudaku & + & - & - & - & - & - \\
\hline Zarda & + & - & + & + & + & - \\
\hline
\end{tabular}

The oral cancers can be classified in to two major categories. The type presenting mainly in Central and South East Asian countries including India, which are considered to be endemic areas for betel quid chewing. The second type presenting mainly in the western world including North America and Europe where the habit of tobacco chewing is not as common as in Asia. In a WHO study, all the products of tobacco and related ingredients are carcinogenic except betel leaves. Few studies have even suggested a protective role of betel leaves in oral cancer. This may be due to a counteraction of chemicals in tobacco and other ingredients by betel leaves. In fact chronic betel chewing is accepted social habit in most of the families in these regions. This also partly explains reduced incidence of oral cancer in females even though the chewing incidence is almost same. 
The common right-handed persons keep the pan over left quid bed; so left sided cancer is more common. Quid bed is the most common site followed by tongue. This is in contrast to western countries, where tongue and oropharyngeal cancers are more common. Etiology and disease biology is completely different. Presence of pathogenic Human Papilloma Virus is one of the etiologies in non-tobacco chewing population ${ }^{(7)}$. Differential disease biology between the two populations has important bearings on the management. Associated changes in the oral cavity like sub mucosal fibrosis, trismus (related to temporo-mandibular joint), multifocal lesions, and sharp and irregular teeth due to lack of oral hygiene and grinding action during tobacco chewing have implication in the management.

Presentation at advanced stage not only increases the burden on multimodality oncological facilities but also reduces the chances of curative management. In the present study $>50 \%$ of patients presented with tumors more than $4 \mathrm{~cm}$ in size and $35 \%$ of patients had gross skin invasion. On the basis of clinical and final histopathological assessment, $61 \%$ of patients had stage IV disease. A recent study from M D Anderson cancer center shows the trends of clinical presentation in the western world. The study comprised a retrospective analysis of 119 patients presented between 1974 and 1993. More than $60 \%$ of patients presented with early stage (T1 and T2) tumors $^{(3)}$. This is true in other studies from western population ${ }^{(2,8)}$, which is converse to our study

Skin and bone infiltration are classified under T4a disease in AJCC staging system. In our experience around $38 \%$ of patients had infiltration of skin with $8 \%$ having Orocutaneous fistula. Growths over the buccal mucosa can grow aggressively within a short span of time, as there are no anatomical structures, which can hinder the growth. Buccinator muscle and buccal pad of fat can easily be infiltrated by the tumors tumor and present in the cheek skin. Getting an adequate margin along the skin is also difficult when there is skin infiltration. The reconstruction of defects following extensive resections is a challenging job; also the adjuvant radiotherapy should be initiated as early as possible in this set of patients.

\section{Conclusion}

Epidemiological features of oral cancer patients vary among different regions. Our study represents the population with predominant tobacco chewing habit, which leads to a particular set of epidemiological features. These simple facts give an insight in to the etiology and disease biology. These facts may be research questions for future studies.

\section{Reference}

1. Globocan 2012 - Home [Internet]. [cited 2017 Sep 9]. Available from: http://globocan.iarc.fr/Default.aspx

2. DeConde A, Miller ME, Palla B, Lai C, Elashoff D, Chhetri D, et al. Squamous cell carcinoma of buccal mucosa: a 40year review. Am J Otolaryngol. 2012 Dec;33(6):673-7.

3. Diaz EM, Holsinger FC, Zuniga ER, Roberts DB, Sorensen DM. Squamous cell carcinoma of the buccal mucosa: one institution's experience with 119 previously untreated patients. Head Neck. 2003 Apr;25(4):267-73.

4. Dwivedi S, Aggarwal A, Singh N, Aggarwal S, Sharma V. Role of family milieu in tobacco addiction: a study in a tertiary-care institution in India. J Health Popul Nutr. 2013 Mar;31(1):130-2.

5. Ghoshal S, Mallick I, Panda N, Sharma SC. Carcinoma of the buccal mucosa: analysis of clinical presentation, outcome and prognostic factors. Oral Oncol. 2006 May;42(5):533-9.

6. Jan J-C, Hsu W-H, Liu S-A, Wong Y-K, Poon C-K, Jiang R-S, et al. Prognostic factors in patients with buccal squamous cell carcinoma: 10-year experience. J Oral 
Maxillofac Surg Off J Am Assoc Oral Maxillofac Surg. 2011 Feb;69(2):396-404.

7. Hübbers CU, Akgül B. HPV and cancer of the oral cavity. Virulence. 2015;6(3):2448.

8. Lubek JE, Dyalram D, Perera EHK, Liu X, Ord RA. A retrospective analysis of squamous carcinoma of the buccal mucosa: an aggressive subsite within the oral cavity. J Oral Maxillofac Surg Off J Am Assoc Oral Maxillofac Surg. 2013 Jun;71(6):1126-31. 